\title{
Insertion of the total artificial heart in the Fontan circulation
}

\author{
Robert D. B. Jaquiss ${ }^{1}$, Ronald K. Woods ${ }^{2}$ \\ ${ }^{1}$ Department of Thoracic and Cardiovascular Surgery, University of Texas Southwestern Medical Center, Children's Health System of Texas, Dallas, \\ TX, USA; ${ }^{2}$ Division of Pediatric Cardiothoracic Surgery, Department of Surgery, Medical College of Wisconsin, Herma Heart Institute, Children's \\ Hospital of Wisconsin, Milwaukee, WI, USA \\ Correspondence to: Robert D. B. Jaquiss, MD. Department of Thoracic and Cardiovascular Surgery, University of Texas Southwestern Medical Center, \\ Children’s Health System of Texas, 1935 Medical District Drive, Dallas, TX 75235, USA. Email: Robert.jaquiss@utsouthwestern.edu.
}

\begin{abstract}
Though the Fontan circulation provides long-term palliation for patients with univentricular hearts, failure of the circulation may ensue, leaving heart transplantation as the only definitive treatment. For Fontan patients awaiting transplant, both "right-sided" and "left-sided" symptoms may be present and severe, hence, biventricular mechanical circulatory support may be indicated. This can be provided by implantation of the total artificial heart (TAH), a procedure which is performed slightly differently than in patients with biventricular hearts. In this article, the unique aspects of implantation of this device in a patient with a Fontan operation are reviewed, with specific attention to the most commonly encountered anatomic variants of importance to the implanting surgeon.
\end{abstract}

Keywords: Total artificial heart (TAH); failing Fontan; univentricular support; Fontan takedown

Submitted Feb 19, 2020. Accepted for publication Mar 19, 2020.

doi: 10.21037/acs.2020.03.11

View this article at: http://dx.doi.org/10.21037/acs.2020.03.11

\section{Introduction}

Surgical palliation for a child born with a functionally univentricular heart consists of a series of procedures culminating in an arrangement whereby all systemic venous blood flows through the lungs passively, i.e., without the addition of hydraulic power normally provided by a subpulmonary ventricle. This arrangement is commonly termed the Fontan circulation since its first successful application was described by Fontan in 1968 (1), and may also be called a total cavopulmonary connection. For most patients, the Fontan circulation is durably effective, providing decades of satisfactory performance. However, in some patients, circulatory insufficiency may develop, a condition termed the "failing Fontan". The manifestations of Fontan failure are generally of two types, somewhat analogous to the manifestations of left heart failure and right heart failure (2). In the former, symptoms are related to inadequacy of the single ventricle, with or without associated atrioventricular or semilunar valve dysfunction, and are those of inadequate systemic cardiac output. In the latter, symptoms are related to inability to achieve adequate pulmonary blood flow at acceptable central venous pressures, and typically include peripheral edema, ascites, pleural effusions, protein-losing-enteropathy (PLE), and more rarely, plastic bronchitis $(\mathrm{PB})$. For completeness, it should be mentioned that the latter two conditions, PLE and $\mathrm{PB}$, may represent a third type of Fontan failure, that related to disorders of lymphatic drainage and connection, exacerbated by elevated central venous pressure (3). It is important to emphasize that the various forms of Fontan failure rarely occur in isolation, and many patients will have some manifestations of left and right heart-type symptoms, occasionally complicated by PLE or PB or both.

For patients whose "Fontan failure" is incompletely responsive to medical therapy, at present the only definitive treatment option is heart transplantation. As with other types of patients with heart failure, the time between listing for heart transplantation and actually receiving an organ is one of great risk for both death and significant clinical deterioration, the latter of which may reduce the likelihood of successful transplant outcome. In patients with biventricular circulation, this risk of death or clinical deterioration may be mitigated with the use of 
mechanical circulatory support as a bridge to transplant, most commonly in the form of left ventricular assist devices (LVADs), now used in between $40 \%$ and $50 \%$ of adult patients undergoing transplantation (4). There has been some limited success with the use of such devices in patients with the Fontan circulation (5). Intuitively, this is most likely to be practical in patients who have the "purest" form of left heart failure, and least likely to be helpful in those with preserved systemic ventricular function, i.e., rightsided failure symptoms. That said, there are case reports of success in both forms of Fontan failure (5). However, for patients with true right and left heart symptoms, mechanical support of both the pulmonary and systemic circulations is desirable. For such patients, the only current FDA-approved device for "biventricular support" is the SynCardia Total Artificial Heart (TAH) (6,7), which is available in two pump sizes (50 and $70 \mathrm{cc}$ ) (SynCardia, Tucson, Arizona, USA). In this paper, we will review the surgical steps necessary for implantation of the TAH in patients with the Fontan circulation.

\section{Operative techniques}

\section{Preparation}

Preparation for TAH implantation in a Fontan patient begins with a complete evaluation of all pathways and connections in the actual Fontan construction, as well as assessing the patency of the central veins, pulmonary arterial tree, pulmonary veins, and the systemic arteries. In addition, the specific morphology of the systemic ventricle(s) and atrioventricular valve anatomy must be understood completely. Computed tomography permits virtual fit testing to determine which pump, if any, will fit the patient (8). It also demonstrates areas of calcification (such as in patch material in the aorta and aortic arch) and any structures at particularly high risk during sternal re-entry. Magnetic resonance imaging provides a reasonable estimate of absolute cardiac output as well as absolute systemic to pulmonary arterial collateral flow. These data have important implications for the conduct of cardiopulmonary bypass during implant (predicting optimal flow rate) and for determining if a maximally operating TAH can provide close to the total cardiac output required for appropriate end-organ perfusion. Finally, it is imperative to determine sites available for peripheral cannulation. In aggregate, this survey will enable the surgeon to plan the conduct of cardiopulmonary bypass to permit implantation of the
TAH as well as to address any "residual" hemodynamic abnormalities such as aortic arch obstruction or branch pulmonary artery stenosis.

\section{Exposition}

\section{Type of Fontan connection}

The Fontan circulation has been most commonly achieved as an "atriopulmonary Fontan", a "lateral tunnel Fontan", or an "extracardiac Fontan" (Figure 1) (9). The operating surgeon must understand surgical details of how the Fontan was achieved, because to perform the TAH implant the Fontan must be "taken down" in order to achieve a site for the connection to the right-sided pump, for both inflow and outflow. An important detail for lateral tunnel patients is to determine whether the second stage operation was performed as a bidirectional Glenn connection or a hemiFontan procedure (10).

\section{Type of ventricular morphology}

Many patients with Fontan circulation will indeed have a univentricular heart, with a single atrioventricular valve. In such patients, attachment of the left-sided inflow "quick connect-atrial cuff" is similar to attachment of the leftside in a patient with a biventricular circulation (11). Other patients with Fontan circulation may actually have two ventricles and even two atrioventricular valves. In such patients, the "extra" ventricle or valve will need to be accounted for in attaching the atrial quick connect. This may involve closure of an atrioventricular valve, resection of the ventricular septum, or other procedure tailored to the unique aspects of patient anatomy.

\section{Operation}

(I) Redo sternotomy and preliminary dissectionall Fontan patients will have had at least one prior sternotomy, and most will have had at least two. Safe sternal re-entry and preliminary dissection are mandatory. In certain cases, it may be prudent to expose peripheral vessels before attempting sternal re-entry, depending on the perceived risk of catastrophic re-entry injury. The first portion of the intrathoracic dissection involves the exposure of the superior and inferior vena cavae, with sufficient length on both to permit cannulation and Fontan 

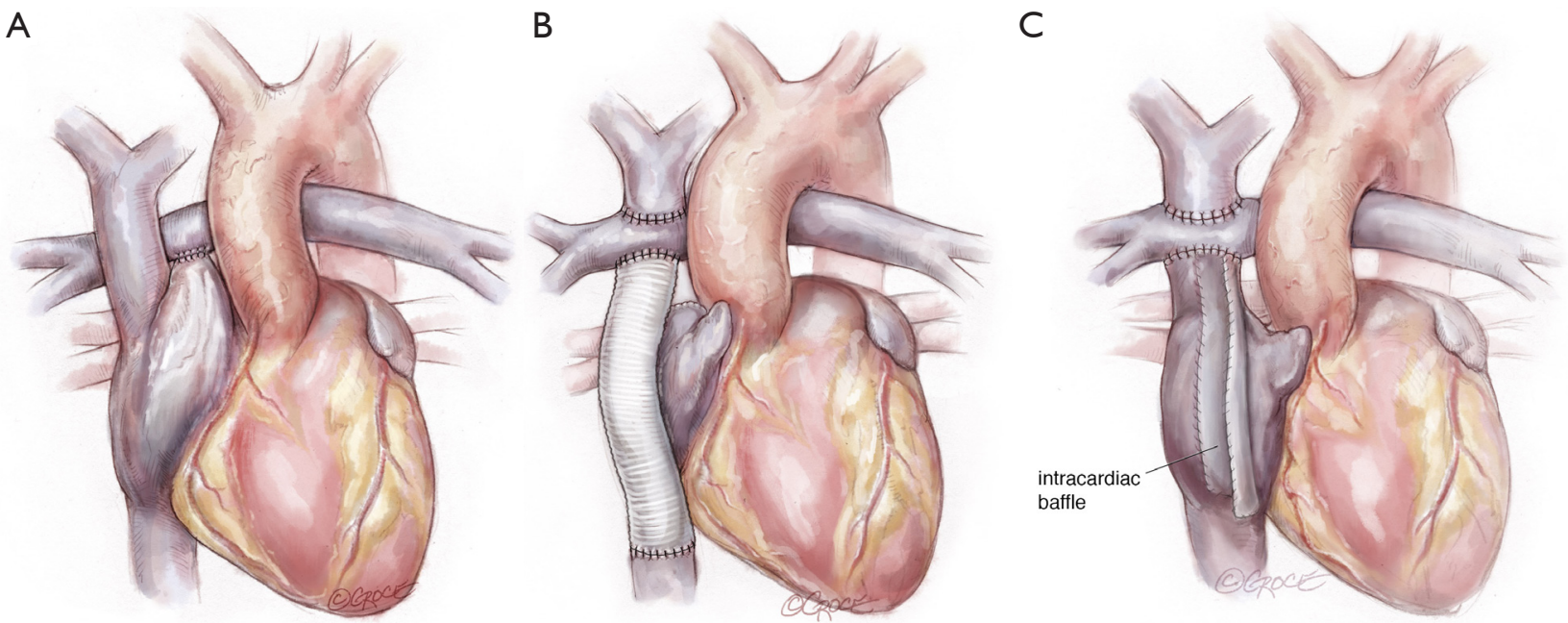

Figure 1 Types of Fontan anatomy. (A) Atriopulmonary Fontan. The superior and inferior vena caval connections to the atrium are intact. The atrial appendage is anastomosed to the pulmonary artery, and there would be no atrial level shunt; (B) extracardiac Fontan. The superior vena cava is anastomosed to the cephalad aspect of the right pulmonary artery. A large diameter graft connected the end of the transected inferior vena cava to the caudal aspect of the right pulmonary artery; (C) lateral tunnel Fontan. The superior vena cava is anastomosed to the cephalad aspect of the right pulmonary artery. The cephalad aspect of the right atrium is anastomosed to the caudal aspect of the right pulmonary artery. A baffle (partition) in the right atrium diverts inferior vena caval blood to the pulmonary artery.

takedown. The central pulmonary artery should also be exposed sufficiently to allow reconstruction after takedown and attachment to the right-sided pump. Likewise, the ascending aorta must be mobilized enough to permit cannulation and crossclamping with sufficient length for anastomosis to the left-sided pump. Ideally, all of this preparation is accomplished off cardiopulmonary bypass. However, patient instability or other factors such as dense adhesions may require cannulation at alternative sites to either complete the dissection or allow for adequate exposure of the inferior vena cava for bicaval venous cannulation.

(II) With preliminary dissection completed, standard aortic and bicaval cannulation is accomplished (alternatively, the innominate vein may be cannulated rather than superior vena cava), with placement of a left-sided vent to capture the pulmonary venous return, which is frequently torrential in Fontan patients as a consequence of systemic to pulmonary collateral artery flow. Early venting is particularly important for the patient with severely diminished ventricular function and a regurgitant neo-aortic valve as fibrillation could lead to acute pulmonary edema. A moderate degree of hypothermia is typically used for both organ preservation and to permit very brief periods of low flow or even circulatory arrest. The aorta is crossclamped to eliminate flow to the myocardium and coronary sinus return.

(III) Fontan takedown is then begun, tailored to the type of Fontan construction. In all cases, the systemic venous circulation will need to be detached from the pulmonary artery, with resultant $\operatorname{defect}(\mathrm{s})$ in the pulmonary artery addressed (Figure 2). In the case of an atriopulmonary Fontan, there will be a single pulmonary arterial defect. This may be patched, or left as the site for connection to the outflow from the right-sided pump. For extracardiac Fontan patients, two defects will result from takedown, and the superior one must be patched. The inferior defect is either patched for used or connection as with the atriopulmonary setup. Management of the lateral tunnel Fontan connection must be individualized, depending on the technique used for the superior cavopulmonary connection (bidirectional Glenn or hemi-Fontan). In the former case, the takedown and reconstruction will be similar to an extracardiac circumstance. In the setting of a hemi-Fontan/lateral tunnel, the pulmonary arterial reconstruction will 

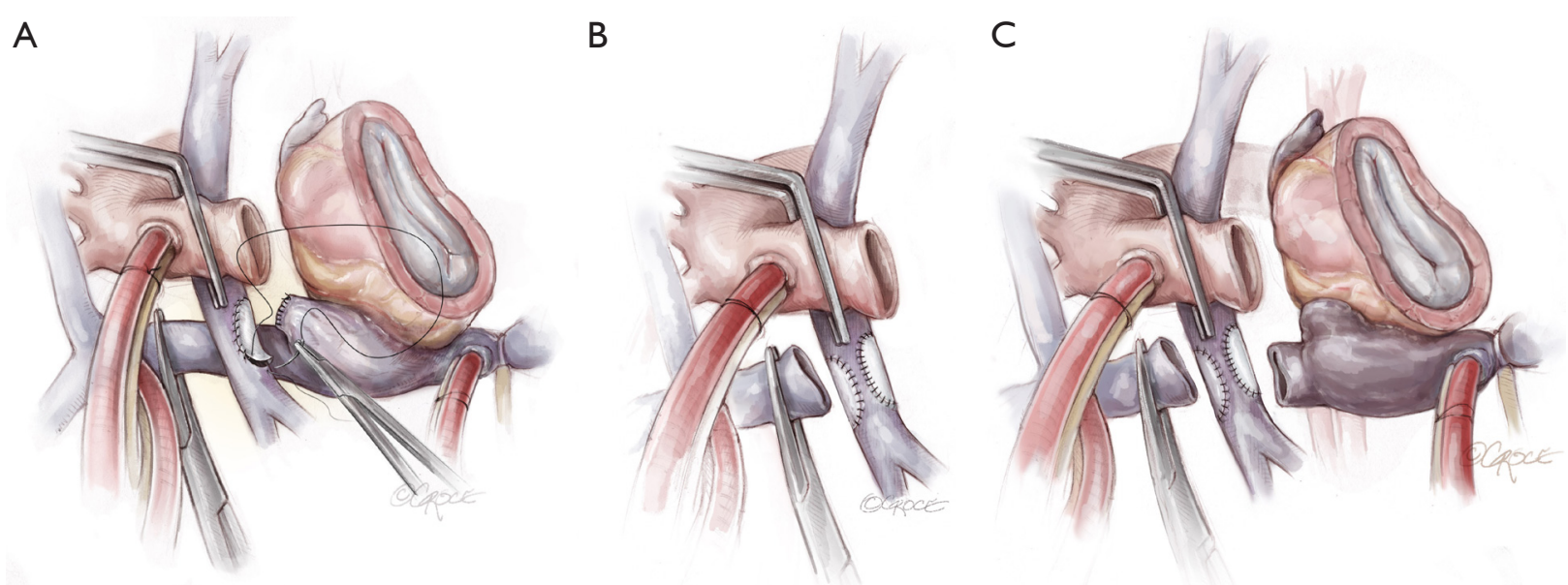

Figure 2 Detaching venous connections form the pulmonary artery. (A) Atriopulmonary Fontan. The atrial appendage is detached from the right pulmonary artery, and the resulting defect is patched. The atrial appendage is closed primarily; (B) extracardiac Fontan. The superior vena cava and extracardiac conduit are detached from the right pulmonary artery and both defects are patched; (C) lateral tunnel Fontan. The superior vena cava and right atrium are detached from the right pulmonary artery and both defects are patched. Note: this assumes that the Fontan was preceded by a bidirectional Glenn. If the Fontan was preceded by a hemi-Fontan (not shown), the takedown of the venous connection will be more extensive and may require additional patch material.

likely be more extensive.

Restoring systemic venous continuity is the next step for patients with lateral tunnel or extracardiac Fontan (Figure 3). In the latter case it is perhaps simplest to excise the original tube graft and to place a new, large diameter polytetrafluoroethylene PTFE graft to connect the superior and inferior vena cavae (6). In the lateral tunnel patient, the SVC can be connected to the original right atrium or superior cavoatrial junction with an interposition PTFE graft. For patients with an atriopulmonary Fontan, wherein both cavae were left attached to the right atrium, this step is not necessary. In all types of Fontan, consideration should be given to eliminating any residual shunts (fenestration or atrial septal defect) at this point, to prevent right to left shunting or paradoxical embolism after TAH implantation.

(V) The systemic ventricle(s) and atrioventricular valve tissue are then excised in order to create a substrate for attachment of the left-sided atrial quick connect (Figure 4). For true single ventricle patients with a single atrioventricular valve, this step is straightforward and analogous to the procedure in a patient with biventricular anatomy (11). For patients with two ventricles, two atrioventricular valves, or both, an alternative solution is required.
In some cases, excision of the second valve to create a single AV orifice may suffice. In other patients complete excision of the ventricular tissue, with attachment of the atrial quick connect to atrial tissue may be the best solution (7). If the atrial chamber is large enough or can be made large enough to be partitioned, attachment of both the left and rightsided quick connects can be made at the atrial level (7). For the $50 \mathrm{cc}$ TAH, atrial quick connect cuffs should not be trimmed excessively, at least for the right-sided pump. The $50 \mathrm{cc}$ TAH is a downsized pump with the angle between the inflow and outflow the same as the $70 \mathrm{cc}$ pump. Cutting the cuff too short could lead to an inadequate space for the right-sided outflow graft to pass over the left-sided outflow graft without posterior effacement.

(VI) The next step is to attach the right-sided inflow quick connect (Figure 5). If an SVC to IVC graft has been inserted, a defect is created in the graft wall, to which the quick connect is anastomosed (6). If there was a lateral tunnel Fontan, a right atriotomy is fashioned, to which the quick connect atrial cuff is anastomosed. If the lateral tunnel channel is relatively small, the edges of the atriotomy may be lengthened with patch material.

(VII) Following this, the outflow grafts are attached to the 

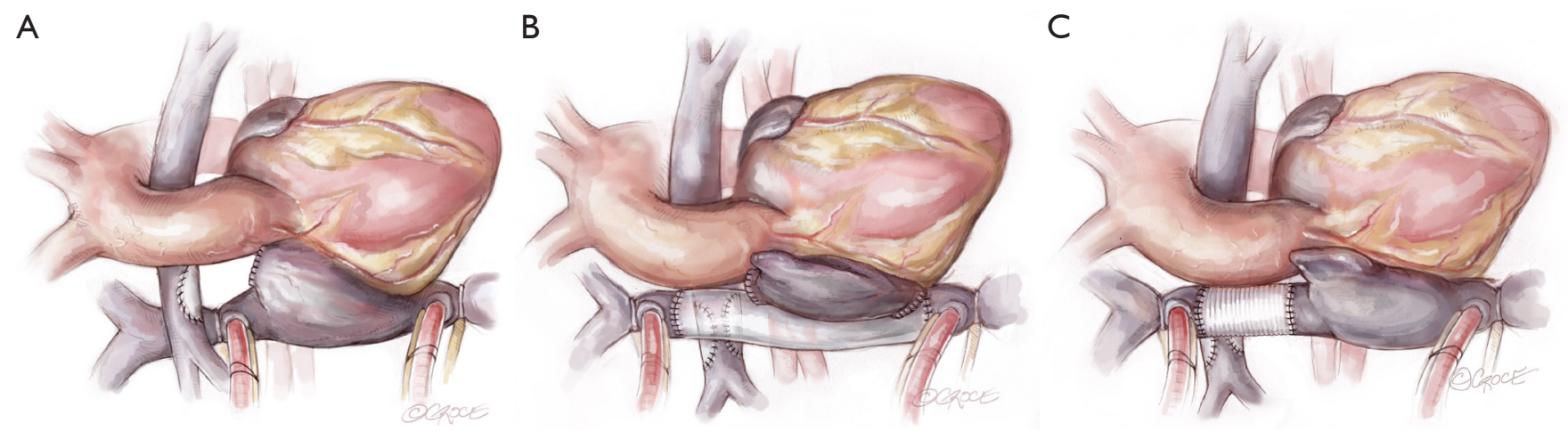

Figure 3 Restoration of venous continuity. (A) Atriopulmonary Fontan. The atrial appendage is detached from the right pulmonary artery, and the resulting defect is patched. The atrial appendage is closed primarily; (B) extracardiac Fontan. The largest diameter PTFE graft, typically $24 \mathrm{~mm}$, is used to connect the superior and inferior vena caval orifices; (C) lateral tunnel Fontan. An appropriately sized PTFE graft is used to connect the superior vena cava to the right atrium.

A

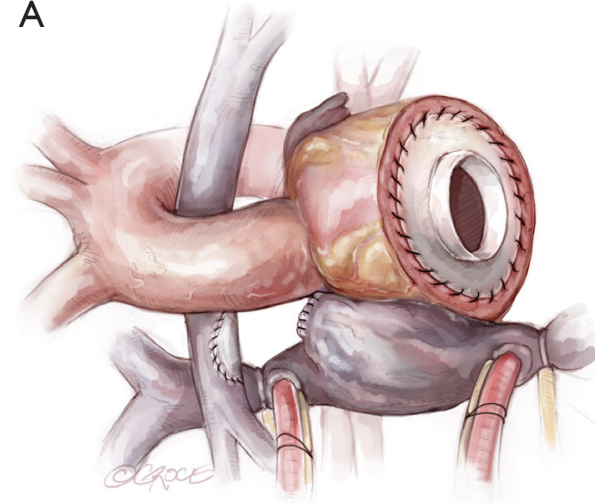

B

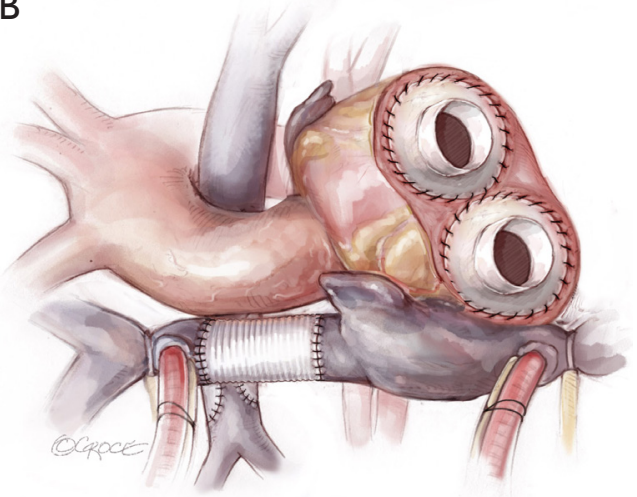

Figure 4 Attachment of the left-sided inflow quick connect. (A) For patients with a single atrioventricular valve. The ventricular mass is excised, and the sewing cuff is anastomosed at the level of the left atrioventricular groove; (B) for patients with two atrioventricular valves and an atriopulmonary or lateral tunnel Fontan. The ventricular mass, atrioventricular groove tissue, and atrial septum are resected. The resultant large common atrium is then septated and the respective left and right inflow quick connects are attached to the neoatria.

aortic remnant and to the pulmonary artery. The latter step must be done with careful planning to ensure that the site of attachment will permit a nondistorted configuration for the right-sided pump. In certain patients with a very short ascending aorta (common in Fontan patients), there will be limited tissue below the aortic clamp. In this case, or if the tissue integrity is poor, the aortic anastomosis may require a period of circulatory arrest.

(VIII) With both inflow and outflow connectors securely anastomosed on each side, left and right pumps are inserted and the drivelines tunneled (11). This portion of the procedure is essentially identical to that for patients with biventricular hearts. After deairing is completed, weaning from bypass should occur uneventfully.

(IX) The final steps in the procedure are the achievement of hemostasis, which must be meticulous, and the preparation for the transplant operation (12). This latter concept will include the judicious placement of PTFE membranes to facilitate reentry and mobilization of the components of the TAH. Purposeful placement of material to facilitate exposure of the vena cavae and great vessels will be 

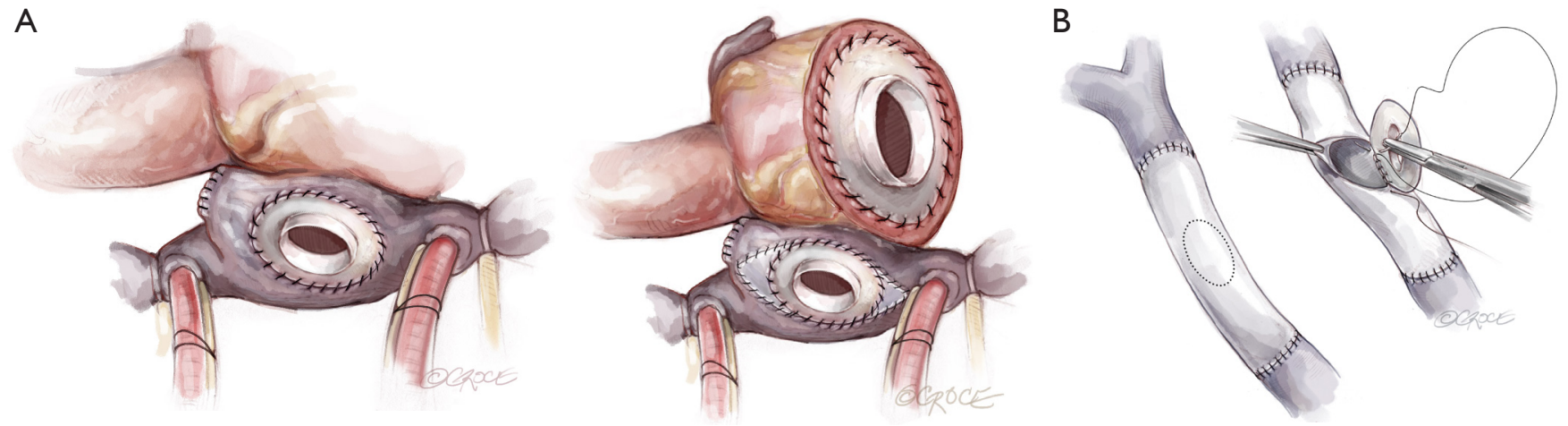

Figure 5 Attachment of the right-sided inflow quick connect. (A) For patients with an atriopulmonary or lateral tunnel Fontan. A longitudinal incision is made in the free wall of the right atrium, to which the quick connect is sewn. In patients with a small right atrium, which is more common in the lateral tunnel arrangement, graft material may be required to augment the right atrial free wall; (B) for patients with extracardiac Fontan. An incision is made in the anterior wall of the new $24 \mathrm{~mm}$ PTFE conduit, to which the right-sided quick connect cuff is anastomosed.

greatly appreciated by the surgeon who performs the subsequent heart transplantation.

(X) Special considerations.

(i) Intraoperative bleeding

While severe bleeding occurs in many types of cardiac surgery, it can be especially troublesome after implanting a TAH in a failing Fontan, in whom the ability of the liver to produce normal levels of clotting factors may impaired. The following are points to consider which may help minimize bleeding-related morbidity.

- Most patients will either have been on warfarin and/or some antiplatelet agent. Both aspects of coagulation should be normalized prior to skin incision.

- Severe post-protamine bleeding requires aggressive product, platelet, and red cell support using a rapid infusion system. Coagulation concentrates and prothrombin complex are useful adjuncts.

- Generous use of hemostatic packing, including securing packing material to the sternal margin tightly with suture, with planned delayed sternal closure is a prudent option in many cases. This avoids excessive bleeding the first night, and packing can be removed the next day without recurrence of bleeding.

(ii) Vasoplegia

Vasoplegia in the Fontan patient shares etiologic aspects with that occurring in other heart failure patients (13), with one additional factor. Extensive systemic to pulmonary collateral flow, which is the rule rather than the exception in Fontan patients, can worsen or solely account for what appears to be vasoplegia. While the usual anti-vasoplegia medications can be used, the best option is to increase device flow. If this does not work, accessible collaterals should be embolized by early cardiac catheterization with the understanding that impact can be difficult to predict. It can range from absolutely no impact to immediate improvement in blood pressure and end-organ perfusion.

\section{Comments}

There is very limited experience with the use of the TAH in Fontan patients, which is doubtless due to the perceived magnitude of the operation as well as the typically severely debilitated state of the candidate patients. The legitimacy of these concerns is attested to by the typically-observed high morbidity and mortality in patients undergoing transplant for failing Fontan. This is not surprising in that the process is appropriately described as performance of a long, complex operation in a debilitated patient who is immediately exposed to immunosuppression. By contrast, support of the failing Fontan patient with the TAH permits the transplant operation to be simpler. Pre-transplant reconstruction is accomplished during device implant, end-organ recovery can begin before exposure to immunosuppression, nutrition 
can be optimized, and rehabilitation can be undertaken. Furthermore, if a patient does not survive the TAH implant, it may be speculated that post-transplant survival would have been even less likely. This, in effect, is the ultimate selection process and may prevent a futile attempt at transplant.

\section{Acknowledgments}

None.

\section{Footnote}

Conflicts of Interest: The authors have no conflicts of interest to declare.

\section{References}

1. Fontan F, Baudet E. Surgical repair of tricuspid atresia. Thorax 1971;26:240-8.

2. Book WM, Gerardin J, Saraf A, et al. Clinical phenotypes of Fontan failure: implications for management. Congenit Heart Dis 2016;11:296-308.

3. Biko DM, DeWitt AG, Pinto EM, et al. MRI evaluation of lymphatic abnormalities in the neck and thorax after Fontan surgery: relationship with outcome. Radiology 2019;291:774-80.

4. Khush KK, Cherikh WS, Chambers DC, et al. The international thoracic organ transplant registry of the international society for heart and lung transplantation: thirty-sixth adult heart transplantation report - 2019; focus theme: donor and recipient size match. J Heart Lung

Cite this article as: Jaquiss RDB, Woods RK. Insertion of the total artificial heart in the Fontan circulation. Ann Cardiothorac Surg 2020;9(2):134-140. doi: 10.21037/acs.2020.03.11
Transplant 2019;38:1056-66.

5. Carlo WF, Villa CR, Lal AK, et al. Ventricular assist device use in single ventricle congenital heart disease. Pediatr Transplant 2017. doi: 10.1111/petr.13031.

6. Rossano JW, Goldberg DJ, Fuller S, et al. Successful use of the total artificial heart in the failing Fontan circulation. Ann Thorac Surg 2014;97:1438-40.

7. Woods RK, Niebler R, Kindel S, et al. A new method for implanting a total artifical heart in the patient with a Fontan circulation. J Thorac Cardiovasc Surg 2019;157:353-5.

8. Ferng AS, Oliva I, Jokerst C, et al. Translation of first north American 50 and $70 \mathrm{cc}$ total artificial heart virtual and clinical implantations: utility of 3D computed tomography to test fit devices. Artif Organs 2017;41:727-34.

9. de Leval MR. The Fontan circulation: a challenge to William Harvey? Nat Clin Pract Cardiovasc Med 2005;2:202-8.

10. Herrmann JL, Brown JW. The superior cavopulmonary connection: history and current perspectives. World J Pediatr Congenit Heart Surg 2019;10:216-22.

11. Arabia FA, Copeland JG, Pavie A, et al. Implantation technique for the CardioWest total artificial heart. Ann Thorac Surg 1999;68:698-704.

12. Ihnken KA, Ramzy D, Esmailian F, et al. Surgical technique to facilitate explantation of mechanical circulatory support devices: LVADs, BiVADs, and TAHs before heart transplantation. ASAIO J 2016;62:211-3.

13. van Vessem ME, Palmen M, Couperus LE, et al. Incidence and predictors of vasoplegia after heart failure surgery. Eur J Cardiothorac Surg 2017;51:532-8. 